\title{
Riesgos asociados a la cirugía en pacientes con problemas cardiacos
}

\author{
Risk associated with surgeries in patients with heart problems
}

\section{Risco associado a cirurgias em pacientes com problemas cardíacos}

\author{
Luz P. Alcívar-Solórzano ${ }^{\mathrm{I}}$ \\ luzmialso@hotmail.com \\ Miguel Á. Arteaga-Intriago II \\ migueluzart@hotmail.com \\ Marco A. Guerrero-Casagualpa \\ marco88_08@hotmail.com \\ María E. Oña-Rivas IV \\ ma.eu.ona.riva@gmail.com \\ Adriano A. Alarcón-Romero $\mathrm{V}$ \\ dr.aalarcon17@gmail.com \\ Jenny E. Landazuri-Barre VI \\ jelb1388@hotmail.com
}

Recibido: 10 de enero de 2019 *Aceptado: 08 de febrero de $2019 *$ Publicado: 05 de abril de 2019

\footnotetext{
Médico General; Asistencial; Hospital Napoleón Dávila Córdoba; Chone, Ecuador.

Médico General; Funciones Hospitalaria del Hospital de Especialidades; Portoviejo, Ecuador.

Médico General Asistencial; Hospital Napoleón Dávila Córdova; Chone, Ecuador.

Médico General; Residente Área de Emergencia Hospital IESS; Portoviejo, Ecuador.

Médico Cirujano; Residente Área de Emergencia Hospital IESS; Portoviejo, Ecuador.

Médico; Residente de Otorrinolaringología y Cirugía Estética Facial en Otorrinos Pichincha, Hospital IESS; Portoviejo, Ecuador
} 


\title{
Resumen
}

El número de procedimientos no cardiacos realizados en pacientes con cardiopatía coronaria está aumentando. A fin de que, para optimizar los resultados, el anestesiólogo debe comprender la fisiología y las implicaciones perioperatorias de la lesión cardíaca, ser capaces de identificar a los pacientes con un alto riesgo de complicaciones anestésicas y comunicación efectiva con los demás profesionales que participan en el cuidado del paciente. Los grupos de mayor riesgo en las complicaciones anestésicas son los lactantes con ventrículo único funcional, los pacientes supra sistémicos con hipertensión pulmonar, pacientes con obstrucción del tracto de salida del ventrículo izquierdo y pacientes con miocardiopatía, además, es fundamental mantenerse actualizado sobre los cambios en el arsenal farmacológico y los avances en tecnología que pueden ser beneficiosos en el cuidado de estos pacientes.

Palabras claves: Problemas Cardiacos; Cirugía; Riesgos Quirúrgicos; Anestesia.

\begin{abstract}
The number of non-cardiac procedures performed in patients with coronary heart disease is increasing. In order to optimize the results, the anesthesiologist must understand the physiology and perioperative implications of cardiac injury, be able to identify patients with a high risk of anesthetic complications and effective communication with the other professionals involved in the procedure. patient care. The groups most at risk in anesthetic complications are infants with functional single ventricle, supra-systemic patients with pulmonary hypertension, patients with obstruction of the left ventricular outflow tract and patients with cardiomyopathy, it is also essential to keep updated on the changes in the pharmacological arsenal and the advances in technology that can be beneficial in the care of these patients.
\end{abstract}

Key words: Cardiac problems; Surgery; Surgical Risks; Anesthesia.

\section{Resumo}

O número de procedimentos não cardíacos realizados em pacientes com doença coronariana está aumentando. Para otimizar os resultados, o anestesiologista deve entender a fisiologia e as implicações perioperatórias da lesão cardíaca, ser capaz de identificar pacientes com alto risco de

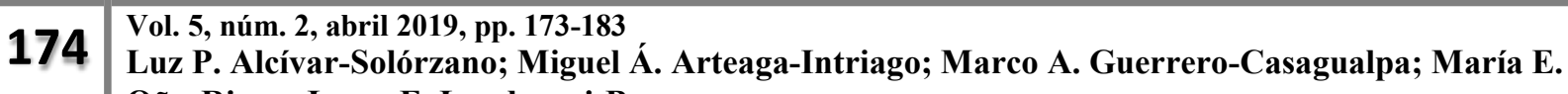
Oña-Rivas; Jenny E. Landazuri-Barre
} 
complicações anestésicas e comunicação efetiva com os demais profissionais envolvidos no procedimento. assistência ao paciente. Os grupos de maior risco em complicações anestésicas são lactentes com ventrículo único funcional, pacientes supra-sistêmicos com hipertensão pulmonar, pacientes com obstrução da via de saída do ventrículo esquerdo e pacientes com cardiomiopatia, também é essencial manter-se atualizado sobre as alterações farmacológicas. arsenal e os avanços tecnológicos que podem ser benéficos no cuidado desses pacientes.

Palavras chave: Problemas cardíacos; Cirurgia; Riscos Cirúrgicos; Anestesia.

\section{Introducción.}

Se informa comúnmente que la incidencia de cardiopatía congénita en los EE.UU. es de aproximadamente 8 por 1000 nacidos vivos. Esto incluye defectos simples y complejos con acceso a cirugía y tratamiento médico, la población infantil y adulta con cardiopatía congénita está creciendo entre 1 y 5\% por año (Roger \& Lloyd-Jones, 2012). Este grupo en crecimiento requiere múltiples procedimientos no cardíacos y atención anestésica asociada y múltiples estudios han demostrado que este grupo tiene un mayor riesgo de complicaciones anestésicas. Revisiones previas de pacientes con enfermedades cardiacas que optan a cirugía no cardíaca se ha centrado en técnica anestésica, agentes farmacológicos y seguimiento posoperatorio.

Una revisión de la literatura actual revela nuevas tendencias interesantes. Posiblemente, el paso preliminar más importante en el cuidado de estos pacientes es el reconocimiento de qué pacientes tienen un alto riesgo de morbilidad y mortalidad relacionada con el procedimiento quirúrgico. Esto es de gran ayuda en el desarrollo de un plan multidisciplinario para el manejo perioperatorio para optimizar la atención y los resultados en el paciente. Además, los equipos de atención de anestesiología deben tener una comprensión profunda de la anatomía, la fisiología y las preocupaciones perianestéticas anticipadas asociadas con lesiones de corazón congénito.

Hay informes de diferentes resultados de diferentes centros que utilizan diferentes técnicas anestésicas y quirúrgicas. Es evidente que el examen institucional de la práctica actual puede llevar a una evolución en el cuidado y mejora en el resultado perioperatorio. 
Esta revisión también hace hincapié en el manejo de la anestesia de una nueva población de pacientes que requieren anestesia para procedimientos no cardíacos, niños con dispositivos de asistencia ventricular. Procedimientos de imagen para pacientes con cardiopatías se utilizan cada vez más para el diagnóstico y planificación quirúrgica. El riesgo de estos procedimientos es casi totalmente anestésico, y las formas en que el riesgo se pueda minimizar son de suma importancia.

El paso preliminar de la atención anestésica a cualquier paciente es la determinación de cuales pacientes están en mayor riesgo. El registro de datos del paro cardíaco perioperatorio pediátrico ha sido muy útil en este sentido. En este informe, el 34\% de los 373 casos de anestesia relacionados a paro cardíaco en pacientes pediátricos con cardiopatía coronaria. Las lesiones más frecuentes en pacientes con arrestos cardiaco fueron ventrículo único, derivación de izquierda a derecha, obstrucción izquierda del tracto de salida ventricular y miocardiopatía.

La menor tasa de mortalidad en pacientes con cardiopatía coronaria tras anestesia fue en aquellos con una derivación de izquierda a derecha. Es importante destacar que el 75\% de los paros cardíacos estaban en pacientes más jóvenes, menores de 2 años (Ramamoorthy, Haberkern, \& Bhananker, 2010).

(van der Griend, 2011) examino la mortalidad postoperatoria en los niños para ayudar a determinar qué pacientes tienen un mayor riesgo anestésico. Esta información ayuda a proporcionar un análisis realista de riesgo / beneficio, y los procedimientos electivos innecesarios pueden abandonarse debido a un riesgo injustificado. Las discusiones multidisciplinarias pueden llevarse a cabo para guiar al personal en la planificación intraoperatoria y postoperatoria. Además, la discusión preoperatoria con la familia y el consentimiento informado debe reflejar no sólo el riesgo del procedimiento, sino también debe abordar el riesgo anestésico. En esta serie, la mitad de las muertes relacionadas con la anestesia fueron pacientes con insuficiencia pulmonar e hipertensión. Estos pacientes son de riesgo extremadamente alto y cualquier procedimiento anestésico debe ser considerado seriamente y tratado con extrema precaución.

\section{Metodología.}

Para el desarrollo de este proceso investigativo, se plantea como metodología la encaminada hacia una orientación científica particular que se encuentra determinada por la necesidad de

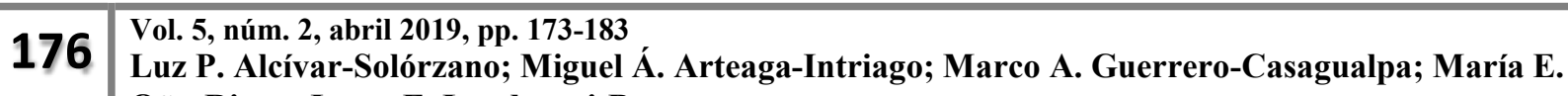
Oña-Rivas; Jenny E. Landazuri-Barre
} 
indagar en forma precisa y coherente una situación, en tal sentido (Davila, 2015) define la metodología "como aquellos pasos previos que son seleccionados por el investigador para lograr resultados favorables que le ayuden a plantear nuevas ideas”.(p.66)

Lo citado por el autor, lleva a entender que el desarrollo de la acción investigativa busca simplemente coordinar acciones enmarcadas en una revisión bibliográfica con el fin de complementar ideas previas relacionadas con el riesgo asociado a la cirugía en pacientes con problemas cardiacos a través de una revisión de literatura, para así finalmente elaborar un cuerpo de consideraciones generales que ayuden a ampliar el interés propuesto.

\section{Tipo de Investigación}

Dentro de toda práctica investigativa, se precisan acciones de carácter metodológico mediante las cuales, se logra conocer y proyectar los eventos posibles que la determinan, así como las características que hacen del acto científico un proceso interactivo ajustado a una realidad posible de ser interpretada. En este sentido, se puede decir, que la presente investigación corresponde al tipo documental, definido por Castro (2016), "se ocupa del estudio de problemas planteados a nivel teórico, la información requerida para abordarlos se encuentra básicamente en materiales impresos, audiovisuales y /o electrónicos". (p.41).

En consideración a esta definición, la orientación metodológica permitió la oportunidad de cumplir con una serie de actividades inherentes a la revisión y lectura de diversos documentos donde se encontraron ideas explicitas relacionadas con los tópicos encargados de identificar a cada característica insertada en el estudio. Por lo tanto, se realizaron continuas interpretaciones con el claro propósito de revisar aquellas apreciaciones o investigaciones propuestas por diferentes investigadores relacionadas con el tema de interés, para luego dar la respectiva argumentación a los planteamientos, en función a las necesidades encontradas en la indagación.

\section{Fuentes Documentales}

El análisis correspondiente a las características que predomina en el tema seleccionado, llevan a incluir diferentes fuentes documentales encargadas de darle el respectivo apoyo y en ese sentido cumplir con la valoración de los hechos a fin de generar nuevos criterios que sirven de referencia a

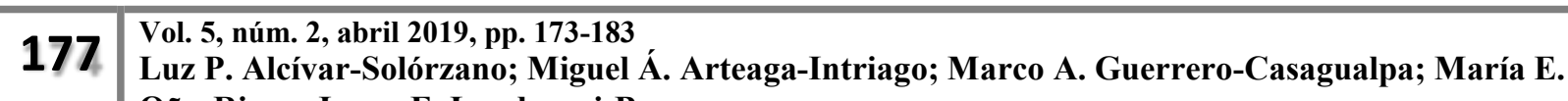
Oña-Rivas; Jenny E. Landazuri-Barre
} 
otros procesos investigativos. Para (CASTRO, 2016) las fuentes documentales incorporadas en la investigación documental o bibliográfica, "representa la suma de materiales sistemáticos que son revisados en forma rigurosa y profunda para llegar a un análisis del fenómeno".(p.41). Por lo tanto, se procedió a cumplir con la realización de una lectura previa determinada para encontrar aquellos aspectos estrechamente vinculados con el tema, con el fin de explicar mediante un desarrollo las respectivas apreciaciones generales de importancia.

\section{Técnicas para la Recolección de la Información}

La conducción de la investigación para ser realizada en función a las particularidades que determinan a los estudios documentales, tiene como fin el desarrollo de un conjunto de acciones encargadas de llevar a la selección de técnicas estrechamente vinculadas con las características del estudio. En tal sentido, (Bolívar, 2015), refiere, que es "una técnica particular para aportar ayuda a los procedimientos de selección de las ideas primarias y secundarias". (p. 71).

Por ello, se procedió a la utilización del subrayado, resúmenes, fichaje, como parte básica para la revisión y selección de los documentos que presentan el contenido teórico. Es decir, que mediante la aplicación de estas técnicas se pudo llegar a recoger informaciones en cuanto a la revisión bibliográfica de los diversos elementos encargados de orientar el proceso de investigación. Tal como lo expresa, (Bolivar, 2015) "las técnicas documentales proporcionan las herramientas esenciales y determinantes para responder a los objetivos formulados y llegar a resultados efectivos" (p. 58). Es decir, para responder con eficiencia a las necesidades investigativas, se introdujeron como técnica de recolección el método inductivo, que hizo posible llevar a cabo una valoración de los hechos de forma particular para llegar a la explicación desde una visión general.

Asimismo, se emplearon las técnicas de análisis de información para la realización de la investigación que fue ejecutada bajo la dinámica de aplicar diversos elementos encargados de determinar el camino a recorrer por el estudio, según, (Bolivar, 2015) las técnicas de procesamiento de datos en los estudios documentales "son las encargadas de ofrecer al investigador la visión o pasos que debe cumplir durante su ejercicio, cada una de ellas debe estar en correspondencia con el nivel a emplear" (p. 123). Esto indica, que para llevar a cabo el procesamiento de los datos

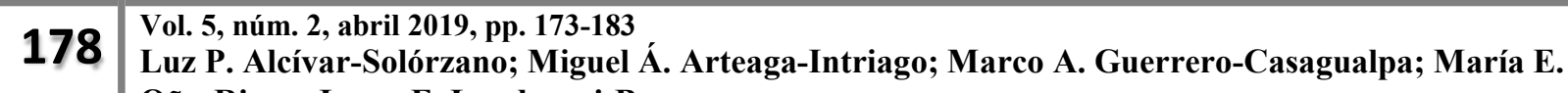
Oña-Rivas; Jenny E. Landazuri-Barre
} 
obtenidos una vez aplicado las técnicas seleccionadas, tales como: fichas de resumen, textual, registros descriptivos entre otros, los mismos se deben ajustar al nivel que ha sido seleccionado.

\section{Resultados.}

El internista frecuentemente es llamado por sus colegas quirúrgicos para una opinión en cuanto al riesgo de anestesia y de un procedimiento quirúrgico en un paciente con cardiopatía orgánica. Una petición de este tipo lleva consigo una serie de preguntas implícitas, entre las más importantes de los cuales se encuentran:

1. ¿Aumenta la anomalía cardíaca el riesgo del procedimiento contemplado?

2. ¿La anestesia y la operación quirúrgica aumentaran las exigencias sobre el corazón más allá de los límites de la reserva cardíaca y por tanto precipitan insuficiencia cardíaca congestiva?

3. ¿La condición cardíaca requiere tratamiento antes de la operación?

4. ¿Es el pronóstico de la enfermedad cardíaca tan grave que la cirugía debe limitarse a un procedimiento de emergencia o paliativo?

5. ¿Es la enfermedad cardíaca de tal naturaleza que lleva consigo la responsabilidad de la muerte súbita durante la anestesia y cirugía?

6. ¿Qué relación tiene el estado del corazón y la elección del agente anestésico?

7. ¿Las complicaciones cardiovasculares deben anticiparse durante la operación y postoperatorio?

La información necesaria para responder estas preguntas por lo general, se pueden obtener de la historia clínica y examen físico sin recurrir a formas de investigación más elaboradas. Todavía se cree con bastante frecuencia que durante la anestesia y las operaciones quirúrgicas el corazón está sujeta a una demanda de trabajo considerablemente mayor, pero no hay evidencia de que este es realmente el caso. Los dos mayores peligros a los que un paciente está expuesto durante una operación bajo anestesia general son la anoxia y el choque. Si estos se evitan con una anestesia adecuada, minimizando la pérdida de sangre y con cuidado en la manipulación de los tejidos, incluso los procedimientos quirúrgicos prolongados y extensos no aumentan la carga en el corazón tanto como con esfuerzo físico moderado. La anestesia competente es rara vez asistida por cianosis o más que alteraciones leves en la respiración, pulso y presión de sangre; y cambios de este tipo no 
se encuentran con más frecuencia en pacientes que tienen cardiopatía orgánica con una reserva miocárdica y coronaria satisfactoria que en individuos normales. Puede ser tomado como una regla general, por lo tanto, que si se evitan la anoxia y el shock, pacientes con cardiopatía orgánica que han sido capaz de llevar a cabo las actividades diarias normales sin experimentar síntomas de miocardio o insuficiencia coronaria puede tolerar anestesia general y cirugía sin más peligros que una persona normal (Christensen, 2012)

Hay algunas excepciones a esta regla, y éstas se mencionarán luego. La presencia de hipertensión no hace afectar la validez de la declaración general, siempre que la función renal no haya sido deprimida en un grado importante. Al estimar el riesgo de anestesia y cirugía en un paciente con cardiopatía orgánica, se debe investigar cuidadosamente la disnea o el dolor subesternal durante el esfuerzo, disnea paroxística nocturna, ataques de insuficiencia coronaria aguda y el ex infarto agudo de miocardio es de mucha más importancia que los hallazgos cardíacos en el examen físico. Ciertos hallazgos, sin embargo, como agrandamiento del corazón, soplos indicativos de daño valvular, galope ritmico, y alteraciones importantes del ritmo del corazón, puede hacer aconsejable cuestionar el paciente de nuevo con el fin de estar seguro de que las preguntas han sido entendidas y con la verdad contestada.

La misma consideración se aplica a la mayoría de las anomalías electrocardiográficas. Con pocas excepciones, un electrocardiograma anormal en un individuo que no ha tenido síntomas de alteración miocárdica o coronaria no indica peligro añadido de anestesia y cirugía. Como ejemplos, pacientes que tienen fibrilación auricular sin la insuficiencia congestiva tolera la anestesia y la operación satisfactoriamente si la frecuencia ventricular ha sido debidamente controlado por digitalis (Duff, deCaen, \& Guerra, 2012).

Ocasionalmente surge una necesidad de anestesia y cirugía en pacientes que sufren insuficiencia cardíaca congestiva. En tales circunstancias es recomendable que haya un período de tratamiento preoperatorio, la intensidad y la duración de los cuales serán determinados por el grado del fracaso y la urgencia de la condición quirúrgica. Una emergencia quirúrgica, como una apendicitis aguda o una úlcera péptica perforada, no permitir la demora, y el riesgo de operación inmediata debe ser aceptado. Si, en una situación de este tipo, el paciente no ha recibido digitalis, una preparación adecuada del medicamento debe ser administrado por inyección intravenosa antes de comenzar la 
anestesia. La dosis usualmente empleado es la mitad o algo más de la cantidad estimada necesaria para completar la digitalización. Después de la operación, inyecciones intravenosas adicionales o intramusculares de cantidades más pequeñas se pueden dar a intervalos de cuatro horas más o menos hasta que se complete el proceso de digitalización. Uno de los diuréticos mercuriales debe administrarse por inyección intramuscular en el mismo tiempo que la dosis inicial de digitalis y posteriormente cada día hasta que la preparación ya no cause diuresis o pérdida de peso. Cuando hay insuficiencia cardíaca congestiva y la condición quirúrgica es de tal tamaño que la operación puede demorarse de manera segura por un poco más de tiempo, la digitalización debe ser realizada por vía oral o, si se encuentran vómitos presentes, por inyección intramuscular de uno de los preparados diseñados para este fin.

Si hay fibrilación articular, la frecuencia ventricular proporciona la guía más útil para determinar si el efecto terapéutico máximo o no de la droga se ha obtenido, se administra ligitalis para reducir la tasa, en ausencia de fiebre, anemia severa y tirotoxicosis, hasta aproximadamente 70 latidos por minuto. Cuando el ritmo normal está presente, la frecuencia cardíaca no ayuda a estimar el grado de digitalización, y entonces se debe prescribir la cantidad del medicamento requerido por el paciente promedio y ser guiado por la respuesta clínica general. El contenido de sodio de la dieta debe ser estrictamente limitado, y si uno de los diuréticos mercuriales debe ser administrado, la cirugía debe posponerse, si es posible, hasta que toda la evidencia del fallo congestivo haya desaparecido. Si esto es hecho, razonablemente se espera que el paciente soporte la anestesia y el funcionamiento sin dificultad, pero si la demora suficiente es imposible y no se puede llevar a cabo un tratamiento adecuado, la cirugía puede ir seguida de un aumento considerable en el grado de fracaso.

Independientemente de la adecuación del tratamiento preoperatorio, pacientes que han tenido falla congestiva antes de la operación debe ser vigilada de cerca durante el período posterior a la cirugía. Complicaciones postoperatorias, como la embolia pulmonar, la atelectasia, la neumonía y la distensión abdominal no están bien soportadas y pueden ser responsable de un rettuni o aumento de la enfermedad.

Las manifestaciones de la descompensación y la mortalidad relacionada con estas complicaciones es considerablemente mayor en pacientes con insuficiencia congestiva antes de la operación que en 
individuos con condiciones normales o en aquellos que tienen enfermedad de corazón orgánico con poco o ningún deterioro de la reserva miocárdica.

\section{Conclusiones.}

La anestesia y las operaciones quirúrgicas no necesariamente incrementan en un alto grado las demandas sobre el corazón en pacientes que tienen enfermedades cardiacas, pero que han sido capaces de continuar sus actividades diarias normales sin síntomas relacionadas a la tolerancia de la anestesia y el funcionamiento sin dificultad siempre que se eviten la anoxia, la hemorragia y el shock. La hipertensión, agrandamiento cardíaco, enfermedad valvular, aparte de la estenosis aórtica avanzada, y las anomalías electrocardiográficas, per se, no incrementan la mortalidad quirúrgica o postoperatoria. Cuando existe insuficiencia miocárdica sintomática o evidencia de insuficiencia cardíaca congestiva, un período de tratamiento preoperatorio con reposo, digitalis, restricción de sodio, y los diuréticos mercuriales son aconsejables.

El tratamiento debe ser lo más completo posible durante el intervalo en el que el retraso de la operación puede ser segura. Los pacientes que han sufrido una falla miocárdica pueden tolerar satisfactoriamente la anestesia y la cirugía. Complicaciones postoperatorias, como neumonía, atelectasia, accidentes tromboembólicos y distensión abdominal, no están bien llevadas, sin embargo, pueden ser responsable de un retorno de la descompensación cardíaca.

Pacientes con fibrilación auricular o aleteo auricular debe digitalizarse antes de la operación, aunque no haya habido síntomas de alteración de la reserva miocárdica ni de la frecuencia ventricular. Se debe evitar la cirugía si es posible en personas con enfermedad coronaria grave.

La anestesia espinal no debe ser empleada en la presencia de estas condiciones. La decisión sobre el tipo exacto de operación y la enfermedad cardíaca orgánica rara vez influye en la realización.

Aunque con frecuencia no ocurren alteraciones del ritmo cardíaco durante la anestesia y el funcionamiento, las complicaciones graves como taquicardia ventricular, parada del corazón y la fibrilación ventricular son infrecuentes. El tratamiento de estas condiciones ha sido discutido. 
Las complicaciones cardíacas postoperatorias no son comunes y rara vez son responsables de la muerte del paciente. La mayor incidencia se produce en pacientes con enfermedad de Iteria córnea. La administración intravenosa de fluidos que contienen sodio debe evitarse durante la operación y el período postoperatorio a menos si su uso está específicamente indicado.

\section{Bibliografía.}

Bolívar, J. (2015). Investigación Documental. México: Pax.

Castro, J. (2016). Técnicas Documentales. México: Limusa.

Christensen, R. (2012). Anaesthetic management and outcomes after noncardiac surgery in patients with hypoplastic left heart syndrome. Eur J Anaesthesio, 425-430.

Davila, A. (2015). Diccionario de Términos Científicos. Caracas: Oasis.

Duff, J., deCaen, A., \& Guerra, G. (2012). Diagnóstico y manejo del paro circulatorio en pacientes con dispositivos de asistencia ventricular pediátrica: presentación de dos casos y pautas sugeridas. Resuscitation.

Ramamoorthy, C., Haberkern, C., \& Bhananker, S. (2010). Anesthesia-related cardiac arrest in children with heart disease. Anesth Analg, 1376-1382.

Roger, V., \& Lloyd-Jones, D. (2012). Heart disease and stroke statistics update: chapter 8. p. 97102.

van der Griend, B. (2011). Postoperative mortality in children after 101885 anesthetics at a tertiary pediatric hospita. Anesth analg, 1440-1447. 